\title{
MicroRNA-490-3p inhibits inflammatory responses in LPS-induced acute lung injury of neonatal rats by suppressing the IRAK1/TRAF6 pathway
}

\author{
GUANG YANG $^{1}$ and YUAN ZHAO ${ }^{2}$ \\ ${ }^{1}$ Department of Pediatrics, Shanxi Medical University, Taiyuan, Shanxi 030001; \\ ${ }^{2}$ Department of Neonatal Internal Medicine, Shanxi Children's Hospital, Taiyuan, Shanxi 030013, P.R. China
}

Received February 21, 2020; Accepted September 8, 2020

DOI: $10.3892 /$ etm.2020.9584

\begin{abstract}
Acute lung injury (ALI) is a main reason for neonatal death. Studying the molecular mechanism behind neonatal ALI is critical for the development of therapeutic strategies. The present study explored microRNA (miR)-490-3p-mediated regulatory effects on lipopolysaccharide (LPS)-induced neonatal ALI. Initially, LPS (10 mg/kg body weight) was injected to 3-8 day old neonatal SD rats to induce ALI, and LPS $(100 \mathrm{ng} / \mathrm{ml})$ was used to treat lung epithelial cells to construct an ALI model in vitro. Next, miR-490-3p, pro-inflammatory factors (that included IL-1 $\beta$, IL-6 and TNF $\alpha$ ), interleukin 1 receptor associated kinase 1 (IRAK1) and TNF receptor associated factor 6 (TRAF6) mRNA expression levels in lung tissues and epithelial cells were assessed via reverse transcription-quantitative PCR. In addition, miR-490-3p mimics were adopted to construct its overexpressed cell model, and Cell Counting Kit-8 and BrdU assays were conducted to assess cell viability. Furthermore, the miR-490-3p target, IRAK was predicted by bioinformatics analysis and verified via Dual-luciferase reporter gene assay. The results revealed that miR-490-3p was markedly downregulated in an LPS-induced rat ALI model, while IL-1 $\beta$, IL-6, TNF $\alpha$, IRAK1 and TRAF6 were all upregulated and negatively correlated with miR-490-3p expression. Moreover, overexpressed miR-490-3p significantly inhibited LPS-induced lung epithelial cell injury and inflammatory response. Mechanistically, miR-490-3p targeted and attenuated IRAK1 expression, which thus inactivated the LPS-mediated TRAF6/NF- $\mathrm{B}$ pathway. Overall, the present study indicated that miR-490-3p overexpression
\end{abstract}

Correspondence to: Dr Yuan Zhao, Department of Neonatal Internal Medicine, Shanxi Children's Hospital, 13 Xinmin North Street, Xinghualing, Taiyuan, Shanxi 030013, P.R. China E-mail: yuao19880811@163.com

Key words: microRNA-490-3p, acute lung injury, inflammatory response, interleukin 1 receptor associated kinase 1 , TNF receptor associated factor 6 significantly inhibited LPS-induced ALI and inflammatory responses by restricting the IRAK1/TRAF6 pathway.

\section{Introduction}

Acute respiratory distress syndrome (ARDS) is one leading cause of neonatal death, which is characterized by excessive inflammation in lung tissues and results in loss of alveolarcapillary membrane integrity and lung dysfunctions $(1,2)$. Previous studies have confirmed that inhibition of inflammatory mediators and oxidative stress alleviates acute lung injury (ALI) (3). Unfortunately, despite significant advances in ALI treatment, the mortality among patients with ARDS reached $20.0 \%$ according to a retrospective cohort study by Killien et al (4) (data from the National Trauma Data Bank). Consequently, it is imperative to study the molecular mechanism of ALI/ARDS and explore new treatment methods.

microRNAs (miRNAs; miRs), typical endogenous short noncoding RNAs, have been revealed to greatly contribute to the regulation of cell proliferation, apoptosis and inflammation (5). Furthermore, miRNAs are considered to be diagnostic biomarkers of certain human inflammations, either promoting or inhibiting inflammatory molecules (6). For example, miR-215-5p has been revealed to be downregulated in sepsisinduced inflammatory damage in $\mathrm{H} 9 \mathrm{c} 2$ cells (7). In addition, miR-214-3p has been revealed to be upregulated in the serum of patients with high-fat diet-induced hyperlipidemic pancreatitis, aggravating tissue damage and inflammation of the accompanied acute kidney injury (8). At present, an increasing number of miRNAs have been found to have a function in ALI. For instance, miR-150 has been revealed to be downregulated in LPS-induced ALI, while overexpressed miR-150 reduced the lung injury (9). In addition, miRNA-1246 has been indicated to repress ALI-induced lung inflammation and apoptosis via $\mathrm{NF}-\kappa \mathrm{B}$ activation and $\mathrm{Wnt} / \beta$-catenin suppression (10). As a novel miRNA, miR-490-3p has been found to regulate the development of various tumors. For example, miR-490-3p suppressed gliomas by inhibiting the expression of the highmobility group AT-hook 2 (11). However, the function and regulatory principal of miR-490-3p in ALI is elusive.

Interleukin 1 receptor associated kinase 1 (IRAK1) and TNF receptor associated factor 6 (TRAF6), as multifunctional 
signal transduction molecules in cells, have been revealed to have a crucial part in the regulation of inflammation by promoting the synthesis and secretion of molecules such as IL-1 $\beta$, IL- 6 and TNF $\alpha(12,13)$. Furthermore, accumulating studies have verified that IRAK1 is regulated by multiple miRNAs. For example, miR-146a has been revealed to regulate gouty arthritis progression by targeting TRAF-6 (14). Overexpressed miR-223 downregulated IRAK-1 in $H$. pylori-infected macrophages, thereby inhibiting the pro-inflammatory response (15). In the present study, bioinformatics analysis (http://starbase.sysu.edu.cn) revealed a potential binding between miR-490-3p and IRAK1. However, whether miR-490-3p regulates the inflammatory response of LPS-induced ALI by targeting IRAK1 remains unknown.

Initially, the present study determined that miR-490-3p was expressed at a low level in LPS-induced ALI, while IRAK1 and TRAF6 were upregulated and their expression was negatively correlated with miR-490-3p. miR-490-3p overexpression has been revealed to markedly inhibit LPS-induced cell injury and inflammation. Furthermore, the present mechanistic experiments demonstrated that miR-490-3p targeted IRAK1 and negatively regulated its expression. Collectively, the present study explored the molecular mechanism of neonatal ALI, with the hope of providing a new theoretical basis for the treatment of this disease.

\section{Materials and methods}

Animal experiments and ARDS induction. Newborn SD rats, (male; age, 3-8 days; weight, 10-15 g; Experimental Animal Center of Shandong University, Jinan, China) were injected with LPS intraperitoneally at $3 \mathrm{mg} / \mathrm{kg}$ to induce ALI $48 \mathrm{~h}$ after LPS administration. The rats were housed at room temperature $\left(18-23^{\circ} \mathrm{C}\right)$ with a $12 \mathrm{~h}$-light/dark cycle. Rats were randomly fed with standard chow and water and adapted to experimental conditions at least 3 days before the experiment. All experiments were carried out following the 'Guidelines for the Use of Laboratory Animal Care' and approved by the Animal Care Use Committee of Shanxi Medical University (Taiyuan, China). All the rats $(n=24)$ were randomly divided into a control and LPS group ( $n=12$ each). The rats were anesthetized by administering intraperitoneal ketamine hydrochloride (60 mg/kg body weight) and xylazine hydrochloride (5 mg-kg body weight) (16-18). LPS (at $10 \mathrm{mg} / \mathrm{kg}$ body weight, E. coli O111:B4; Sigma-Aldrich; Merck KGaA) was dissolved in $20 \mu \mathrm{l}$ sterile phosphate-buffered saline (PBS) for $24 \mathrm{~h}$ and then injected intratracheally. The control group received the same volume of PBS. The health and behaviors of rats were monitored every $12 \mathrm{~h}$. Before the rats were sacrificed to collect the lung tissues, the rats were placed in a closed container with a low concentration of $\mathrm{CO}_{2}(10-30 \%)$. Once the rats lost consciousness under the low concentration of $\mathrm{CO}_{2}$ environment, they were euthanized with $100 \% \mathrm{CO}_{2}$ (the experiment was carried out in September 2019). The euthanasia was confirmed when lack of respiration and color fading of eyes were observed in the rats for a minimum of $1 \mathrm{~min}$. All efforts were made to minimize their suffering. Preemptive euthanasia was performed for humane reasons if rats exhibited any of the following signs: Emaciated, gasping, no response to touch or an anal temperature $<25^{\circ} \mathrm{C}$.
Immunohistochemistry. Clinical specimens were fixed with $10 \%$ formaldehyde at room temperature for $24 \mathrm{~h}$ and embedded in paraffin, then the sections $(4 \mu \mathrm{m})$ were dewaxed and hydrated. Firstly, dewaxed sections were dried at $37^{\circ} \mathrm{C}$ for $2 \mathrm{~h}$, and its endogenous peroxide was blocked with $1 \% \mathrm{H}_{2} \mathrm{O}_{2}$ for $5 \mathrm{~min}$. Subsequently, the sections were washed 3 times with PBS, blocked with 5\% BSA (cat. no. ST025; Beyotime Institute of Biotechnology) for $1 \mathrm{~h}$ at room temperature, and then incubated with anti-IRAK1 antibody (1:200; cat. no. ab238; Abcam) at $4^{\circ} \mathrm{C}$ overnight. Subsequently, they were washed with PBS and incubated with goat anti-rabbit IgG H\&L (HRP) secondary antibody (1:500; cat. no. ab205718; Abcam) for $1 \mathrm{~h}$ at room temperature. The sections were rinsed again and stained with 3,3-diaminobenzidine hydrochloride for $1 \mathrm{~min}$. Finally, they were rinsed with double-distilled water and stained with hematoxylin and eosin (H\&E) for $1 \mathrm{~min}$ at room temperature, and observed under a light microscope (magnification, x200).

Cell culture and treatments. The A549 cell line (cat. no. CCL-185 ${ }^{\mathrm{TM}}$ ) was obtained from American Type Culture Collection (ATCC) and HPAEpiC (cat. no. 3200) (human type II alveolar epithelial cell) was purchased from ScienCell Research Laboratories, Inc. These two cell lines were incubated in Roswell Park Memorial Institute (RPMI-1640) medium (Gibco; Thermo Fisher Scientific, Inc.) supplemented with $10 \%$ fetal bovine serum and $1 \%$ antibiotic antifungal solution (both from Sigma-Aldrich; Merck KGaA). In addition, the cells were cultured in an incubator with humidified $\mathrm{CO}_{2}$ at $37^{\circ} \mathrm{C}$. miR-490-3p mimics (5'-CAACCUGGAGGACUCCAUGCUG-3') and its negative control (miR-NC, 5'-ACCGCUAAUCAUACGAAUACAC-3') were obtained from Shanghai GenePharma Co., Ltd. The cells $\left(1 \times 10^{5}\right)$ were seeded in 12-well plates and transfected with miR-490-3p or miR-NC (50 pmoles) using Lipofectamine ${ }^{\circledR}$ 2000 (Invitrogen; Thermo Fisher Scientific, Inc.) at $37^{\circ} \mathrm{C}$ for $24 \mathrm{~h}$ according to the manufacturer's instructions. Cells were used for subsequent experiments after $24 \mathrm{~h}$. Four groups were designed, namely the control group, the LPS group, the LPS + NC group and the LPS + miR-490-3p. LPS (100 ng/ml) was used to treat the cells for $4 \mathrm{~h}$.

Reverse transcription-quantitative PCR (RT-qPCR). Total RNA was extracted from the cells using TRIzol ${ }^{\circledR}$ reagent (Thermo Fisher Scientific, Inc.) and reverse-transcribed into cDNA with a cDNA Synthesis Kit (Sangon Biotech Co., Ltd.) according to the manufacturer's protocol. RT-qPCR was conducted with a SYBR Prime Script RT-PCR kit (Invitrogen; Thermo Fisher Scientific, Inc.). The thermocycling conditions were as follows: $10 \mathrm{~min}$ at $95^{\circ} \mathrm{C} ; 40$ cycles of $1 \mathrm{~min}$ at $95^{\circ} \mathrm{C}, 2 \mathrm{~min}$ at $63^{\circ} \mathrm{C}$ and $1 \mathrm{~min}$ at $72^{\circ} \mathrm{C}$. The primers were synthesized by Sangon Biotech Co., Ltd. The miRNA relative expression was calculated by normalizing to U6 small nuclear RNA. The primer sequences were as follows: miR-490-3p forward, 5'-CGTGGATCCTTCTTCA ACCAACGGTGGTG-3' and reverse, 5'-CCAGAATT CAAAGCAGGA AGAGTAAGACTTCC-3'; IRAK1 forward, 5'-CCTCCAGGTTCCACTCTCTG-3' and reverse, 5'-A ACCACCCTCTCCAATCCTG-3'; TRAF6 forward, 5'-TTGCACATGAGACTGTTGGC-3' and reverse, 5'-CTTCGAATGGTCCGCTTGAG-3'; GAPDH forward, 
5'-AACGGATTT GGTCGTATTG-3' and reverse, 5'-GGAA GATGGTGATGGGATT-3'; U6 forward, 5'-GGAGCGAG ATCCCTCCAAAAT-3' and reverse, 5'-GGCTGTTGTCAT ACTTCTCATGG-3'. Relative expression was calculated using the $2^{-\triangle \triangle C q}$ method (19), using U6 or GAPDH as normalization controls.

Western blot analysis. RIPA lysate (Roche Diagnostics) was used to isolate the total proteins from both lung tissues and cells. Protein concentration was determined using a BCA kit (Beyotime Institute of Biotechnology). A total of $50 \mu \mathrm{g}$ of protein was loaded on a $12 \%$ polyacrylamide gel and electrophoresed at $100 \mathrm{~V}$ for $2 \mathrm{~h}$ and electrically transferred to polyvinylidene fluoride (PVDF) membranes. After being blocked with 5\% skimmed milk (room temperature; $1 \mathrm{~h}$ ), the membranes were washed 3 times with TBS- $0.1 \%$ Tween for 10 min each time, then incubated with antibodies of anti-IRAK1 (1:1,000; cat. no. ab238), anti-TRAF6 (dilution 1:1,000; cat. no. ab227560), anti-phosphorylated (p-)NF- $\mathrm{KB}$ (dilution 1:1,000; cat. no. ab76302) and anti-NF- $\mathrm{KB}$ (dilution 1:1,000; cat. no. ab32536; all from Abcam) at $4^{\circ} \mathrm{C}$ overnight. Then, the membranes were washed with TBST again and incubated with goat anti-rabbit IgG H\&L (HRP) (dilution 1:3,000; cat. no. ab205718; Abcam) at room temperature for $1 \mathrm{~h}$. Subsequently, the membranes were washed 3 times, 10 min each with TBST. Finally, Pierce ${ }^{\mathrm{TM}}$ ECL Western Blotting Substrate (Invitrogen; Thermo Fisher Scientific, Inc.) was used for imaging, and ImageJ (v1.48; National Institutes of Health) was adopted to semi-quantify the gray value of each protein.

Cell Counting Kit-8 (CCK-8) assay. Cells of each group in the logarithmic growth phase were trypsinized, centrifuged $(500 \mathrm{x} \mathrm{g})$ for $5 \mathrm{~min}$ at room temperature and counted, and then inoculated in $96-$ well plates at $2 \times 10^{3}$ cells/well. After $24 \mathrm{~h}$ of incubation (at $37^{\circ} \mathrm{C}$ with $5 \% \mathrm{CO}_{2}$ ), the culture solution was removed. After treatment according to the experimental group, $10 \mu \mathrm{l}$ of CCK-8 solution (Beyotime Institute of Biotechnology) was added to each well and incubated at $37^{\circ} \mathrm{C}$ for $1 \mathrm{~h}$. A microplate reader was used to detect the absorbance value of each well at $450 \mathrm{~nm}$. Four parallel wells were set in each group, and each experiment was repeated 3 times.

BrdU assay. The cells were seeded in a 12 -well plate at $1 \times 10^{5}$ cells/well and incubated at $37^{\circ} \mathrm{C}$ with $5 \% \mathrm{CO}_{2}$ in a humidified environment for $12 \mathrm{~h}$ until fully adherent. After treating the cells of each group, BrdU at a final concentration of $30 \mu \mathrm{mol} / 1$ was added and incubated at $37^{\circ} \mathrm{C}$ for $4 \mathrm{~h}$. Subsequently, the cell slides were washed with PBS and fixed with $4 \%$ formaldehyde at room temperature for $10 \mathrm{~min}$, and then acidified and denatured in PBST (PBS with $0.1 \%$ Tween-20) containing $2 \mathrm{~mol} / \mathrm{l}$ of hydrochloric acid for $30 \mathrm{~min}$. After being blocked with PBST containing 5\% bovine serum albumin (Beyotime Institute of Biotechnology) for $1 \mathrm{~h}$, the cells were incubated with BrdU antibody (1:1,000 in PBS; cat. no. ab8152; Abcam) for $2 \mathrm{~h}$ at room temperature. Then the cells were washed 3 times with PBS, and incubated with goat anti-mouse IgG H\&L (Alexa Fluor $^{\circledR}$ 647; cat. no. ab150115; Abcam) (1:1,000 in PBS) for $1 \mathrm{~h}$. Finally, DAPI (Beyotime Institute of Biotechnology) was used for labeling the nuclei, and BrdU was observed with a fluorescence microscope (magnification, x200). The cell proliferation rate was calculated as follows: Cell proliferation rate $=$ Number of positive cells/number of total cells $\times 100 \%$. The experiment was repeated three times.

Enzyme-linked immunosorbent assay (ELISA). The supernatant of cell culture media was collected and centrifuged on a low-speed centrifuge $(500 \mathrm{x} \mathrm{g})$ for $5 \mathrm{~min}$ at room temperature. Then the expression of IL-6, TNF $\alpha$ and IL-1 $\beta$ in the supernatant was detected using ELISA kits for IL-6 (cat. no. 70-EK206/3-96), TNF- $\alpha$ (cat. no. 70-EK282/3-96) and IL-1 $\beta$ (cat. no. 70-EK201B/3-96) according to the manufacturer's instructions. All of the detection kits were obtained from Hangzhou Multi Sciences (Lianke) Biotech, Co., Ltd.

Luciferase reporter assay. Bioinformatic analysis was conducted to predict the downstream target of miR-490-3p using StarBase v3.0 (http://starbase.sysu.edu.cn) (20). The amplified DNA sequence was cloned into pmirGLO Dual-luciferase vectors (Promega Corporation) to construct wild-type (WT) IRAK1 3'-untranslated region (UTR) and mutant (MUT) IRAK1 3'-UTR reporter vectors. A549 cells $\left(1 \times 10^{5}\right)$ were seeded in 24 -well plates overnight and then transfected with IRAK1-WT or IRAK1-MUT reporter vector and transfected with miR-490-3p mimics or negative controls using Lipofectamine ${ }^{\circledR} 2000$ (Invitrogen; Thermo Fisher Scientific, Inc.). A Dual-luciferase reporter gene assay system (Promega Corporation) was adopted to determine luciferase viability $48 \mathrm{~h}$ after transfection. Renilla luciferase activity was used as the internal control.

Statistical analysis. SPSS (version 20.0; IBM Corp.) was used for data analysis. All data were expressed as the mean \pm SD. Statistical analysis was performed using an unpaired Student's t-test. Differences between the two groups were analyzed using $\chi^{2}$. The correlation of the molecules was analyzed using Pearson's correlation test. $\mathrm{P}<0.05$ was considered to indicate a statistically significant difference.

\section{Results}

miR-490-3p is decreased in LPS-induced ALI. To probe miR-490-3p function in ALI, an LPS-induced ALI rat neuronal model was established and the pathological alteration in lung tissue of LPS rats was examined using H\&E staining (Fig. 1A). As a result, it was revealed that there was edema, lung tissue alteration, incomplete nuclear staining, unclear cell boundaries and accumulating inflammatory cell and neutrophil infiltration in the lungs of the LPS-treated rats (Fig. 1A). In addition, RT-qPCR revealed that miR490-3p was downregulated in LPS-induced ALI (Fig. 1B). Furthermore, it was also revealed that the inflammatory factors IL-1 $\beta$, IL- 6 and TNF $\alpha$ were significantly upregulated in the lung tissues of LPS-induced ALI compared with the control group (Fig. 1C). Furthermore, Pearson correlation analysis revealed that miR-490-3p was negatively correlated to IL-1 $\beta$, IL- 6 and TNF $\alpha$ expression in LPS-induced ALI (Fig. 1D). The aforementioned findings indicated that miR-490-3p was downregulated in LPS-induced ALI, and inversely related to the inflammatory response. 
A
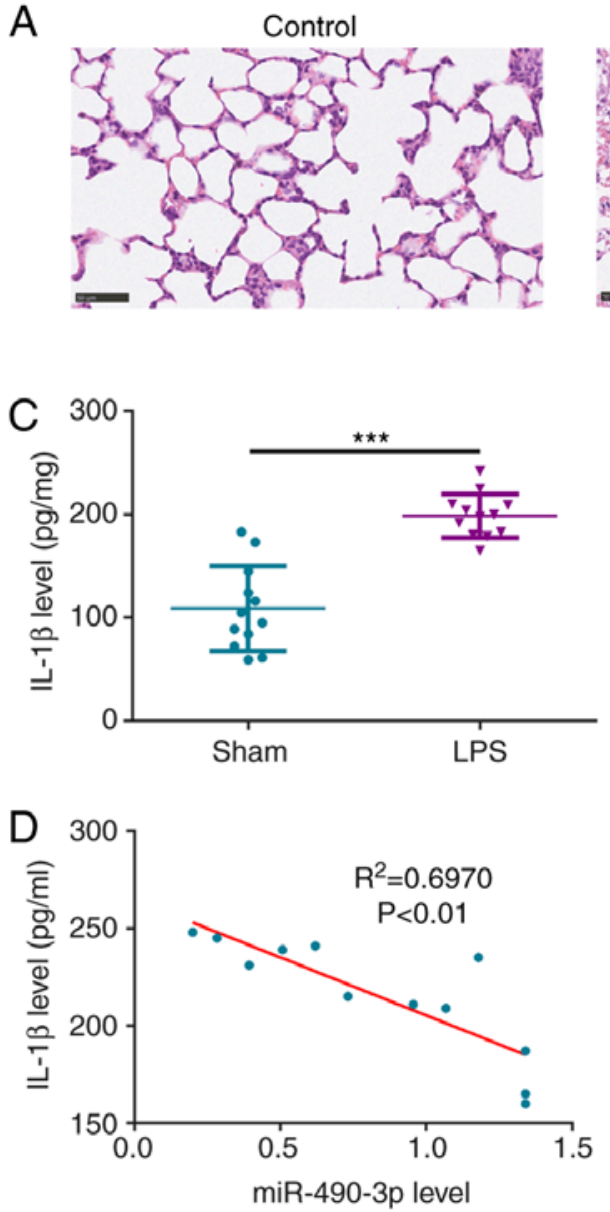

LPS
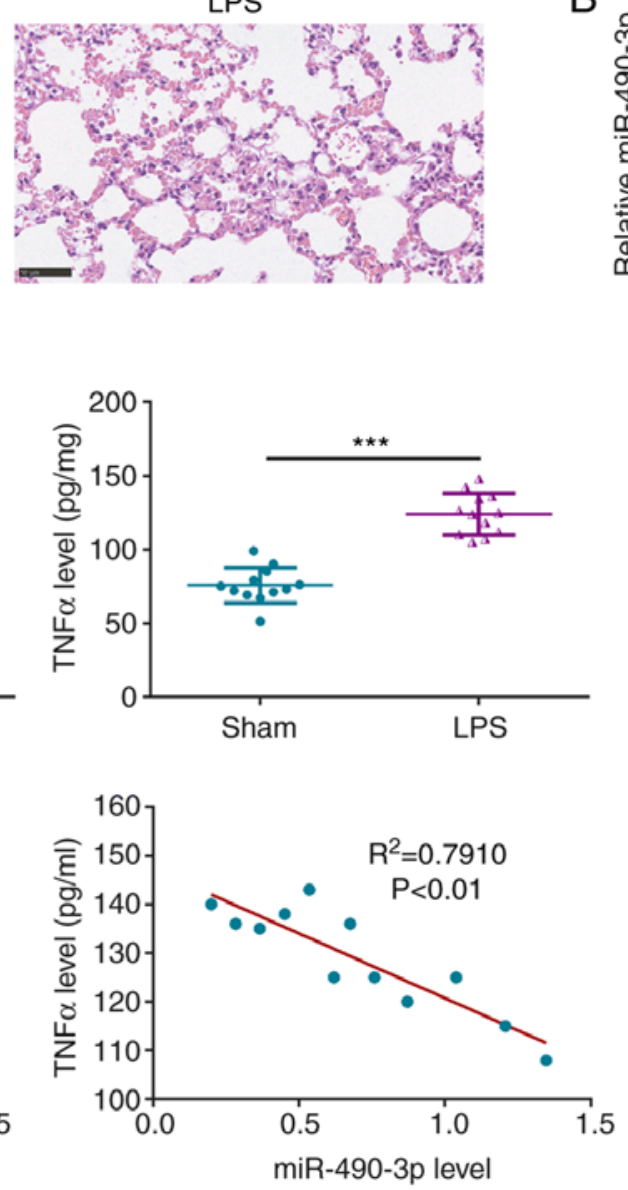

B
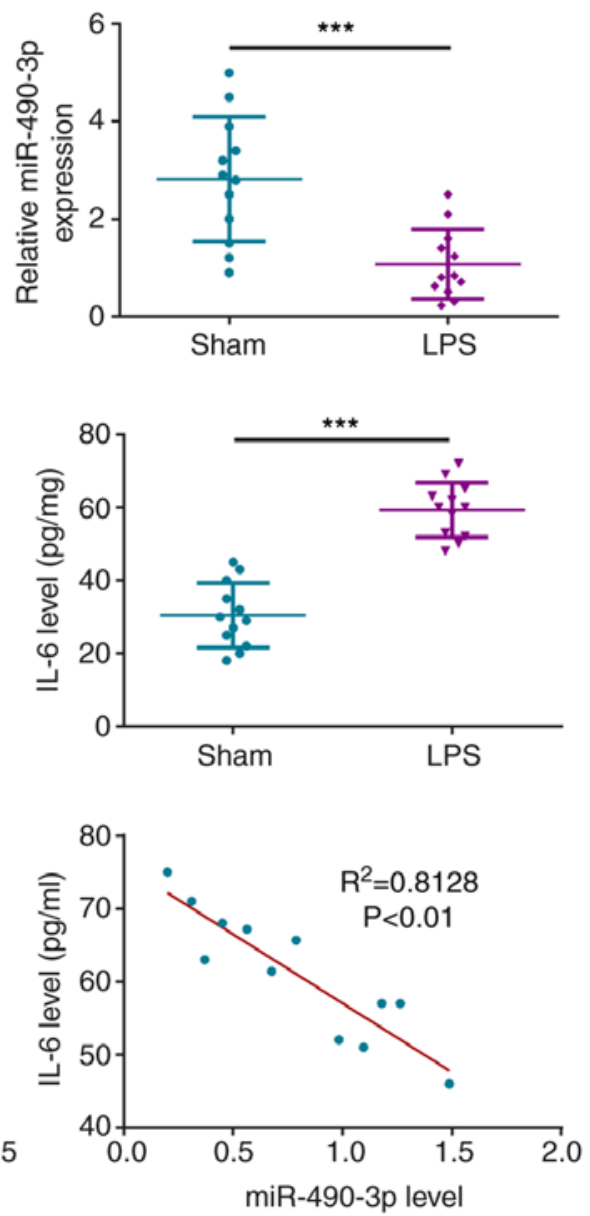

Figure 1. miR-490-3p is downregulated in LPS-induced ALI. (A) Pathological alteration in the lung tissues of LPS-induced ALI rats were detected by hematoxylin and eosin staining. (B) Reverse transcription-quantitative PCR was performed to examine miR-490-3p relative expression in the lung tissues. (C) The inflammatory factors IL-1 $\beta$, IL-6 and TNF $\alpha$ in the control group and LPS-induced ALI rats were examined by ELISA. (D) Correlation among IL-1 $\beta$, IL-6, TNF $\alpha$ and miR-490-3p was analyzed by Pearson correlation tests. ${ }^{* * *} \mathrm{P}<0.001$. LPS, lipopolysaccharide; ALI, acute lung injury; miR, microRNA.

IRAK1 and TRAF6 are upregulated in ALI and negatively correlated with miR-490-3p. The relative expression of IRAK1 and TRAF6 in various groups was examined by RT-qPCR. The results indicated that both IRAK1 and TRAF6 mRNAs were significantly upregulated in LPS-induced ALI (Fig. 2A). In addition, it was confirmed that miR-490-3p was negatively correlated with IRAK1 and TRAF6 expression (Fig. 2B). Moreover, immunohistochemistry indicated that the number of IRAK1-positive cells were increased in LPS-induced ALI (Fig. 2C). Furthermore, western blot analysis demonstrated that the expression of IRAK1, TRAF6 and $\mathrm{p}-\mathrm{NF}-\kappa \mathrm{B}$ proteins in LPS-induced ALI was significantly enhanced compared with that of the control group (Fig. 2D and E). These studies indicated that IRAK1 and TRAF6 were upregulated in ALI and were inversely related to $\mathrm{miR}-490-3 \mathrm{p}$.

miR-490-3p overexpression distinctly inhibits LPS-induced cell injury and inflammatory response. $\mathrm{miR}-490-3 \mathrm{p}$-overexpressed A549 cells were constructed to further investigate its mechanism in LPS-induced ALI (Fig. 3A). RT-qPCR revealed that miR-490-3p was increased in the LPS + miR-490-3ptreated group compared with the LPS + NC-treated group (Fig. 3B). Moreover, cell proliferation of LPS + miR-490-3p was enhanced compared with the LPS + NC-treated group
(Fig. 3C). Furthermore, BrdU assays affirmed that the cell viability of the LPS + miR-490-3p group was significantly increased compared with the LPS + NC group (Fig. 3D). ELISA assays also indicated that the inflammatory factors IL-1 $\beta$, IL-6 and TNF $\alpha$ were decreased in the LPS + miR-490-3p-treated group compared with the LPS + NC group (Fig. 3E). These studies indicated that miR-490-3p overexpression significantly inhibited LPS-induced cell injury and inflammatory response.

miR-490-3p overexpression suppresses IRAK1 and TRAF6 expression. To further study the miR-490-3p mechanism in LPS-induced ALI, RT-qPCR and western blot analysis was used to examine the relative expression of IRAK1, TRAF6 and $\mathrm{p}-\mathrm{NF}-\kappa \mathrm{B}$. It was revealed that the mRNA and protein expression of IRAK1, TRAF6 and p-NF- $\kappa \mathrm{B}$ were significantly downregulated in the LPS + miR-490-3p treatment group when compared with the LPS + NC treatment group (Fig. 4A-D). As a result, miR-490-3p appeared to inhibit the LPS-activated IRAK1/TRAF6/NF- $\mathrm{B}$ pathway.

miR-490-3p targets IRAK1. To identify the downstream molecular mechanism of miR-490-3p, its candidate targets were analyzed via StarBase (http://starbase.sysu.edu.cn). The results indicated that miR-490-3p could bind to IRAK1 

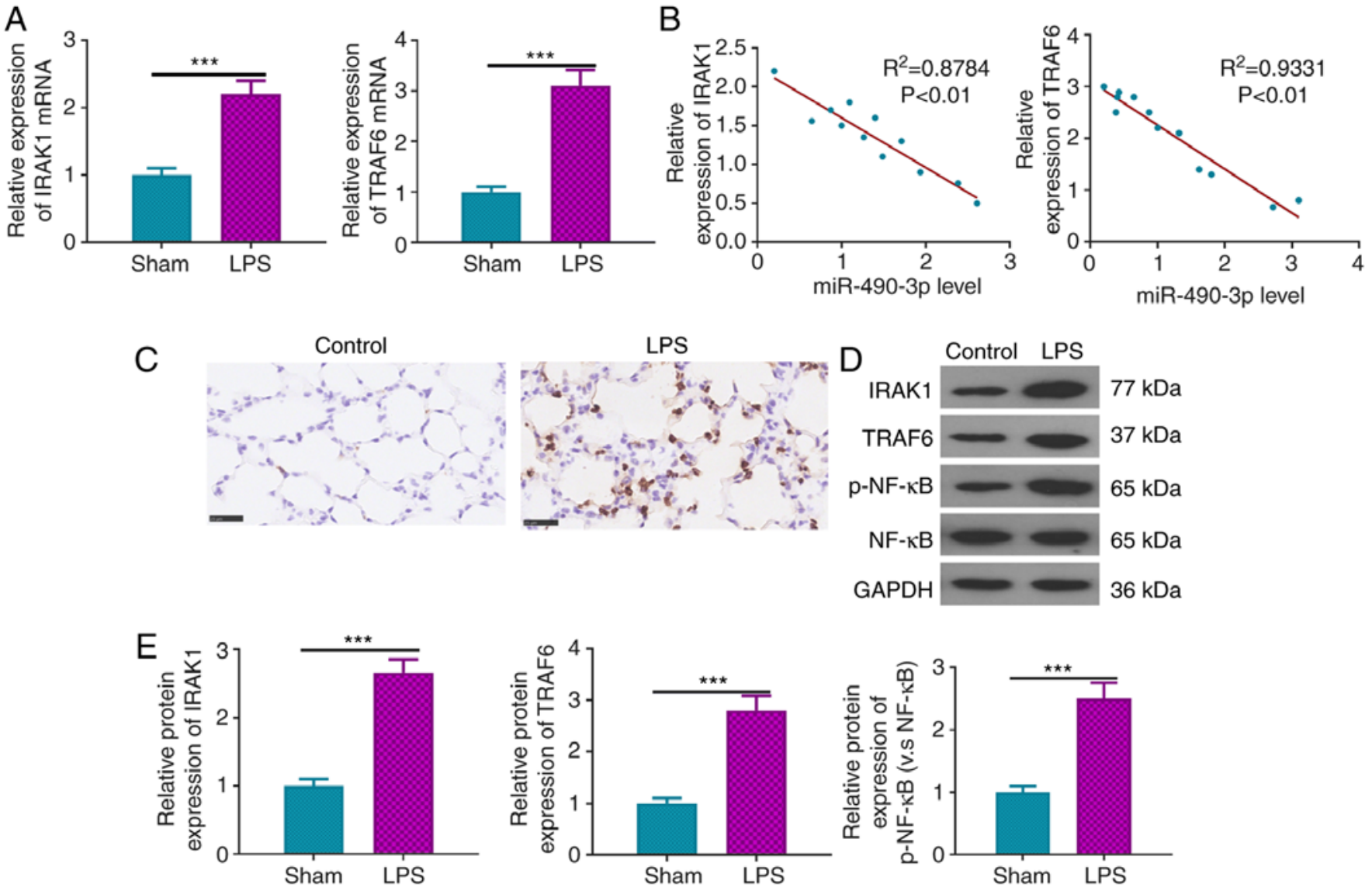

Figure 2. IRAK1 and TRAF6 are upregulated in ALI and negatively correlated with miR-490-3p (A) Reverse transcription-quantitative PCR was adopted to monitor the IRAK1 and TRAF6 relative expression in the control group and LPS-induced ALI. (B) The correlations among miR-490-3p, IRAK1 and TRAF6 in LPS-induced ALI was analyzed. (C) Immunohistochemical staining was used for detecting IRAK1 expression in lung tissues. The relative expression levels of IRAK1, TRAF6, p-NF- $\mathrm{KB}$ in the control group and LPS-induced ALI lung tissues were assessed by (D) western blot analysis and subsequently (E) semiquantified. ${ }^{* * * *} \mathrm{P}<0.001$. IRAK1, interleukin 1 receptor associated kinase 1; TRAF6, TNF receptor associated factor 6; ALI, acute lung injury; miR, microRNA; LPS, lipopolysaccharide; p-, phosphorylated.

(Fig. 5A). Similarly, the targeted binding relationship between miR-490-3p and IRAK1 was verified using a dual luciferase reporter assay. miR-490-3p mimics were revealed to attenuate the luciferase viability of IRAK1-WT but had no significant effects on that of the IRAK1-MUT (Fig. 5B). Thus, these results verified that miR-490-3p decreased LPS-induced inflammation by directly targeting IRAK1.

\section{Discussion}

Neonates are particularly susceptible to ALI, which is one of the most frequent causes of mortality in newborns (21). As the main pathogenic component of Gram-negative bacteria, LPS has been indicated to be an important cause of ALI (22). LPS causes the activation of related inflammatory cells, thus releasing IL-1 $\beta$, IL-6, TNF $\alpha$ and other inflammatory mediators, which results in diffuse injuries of alveolar epithelial cells, alveolar capillary endothelial cells and pulmonary interstitium $(1,23)$. Consequently, studying the pathogenesis of ALI has a direct guiding significance for its treatment. Initially, miR-490-3p was revealed to be significantly downregulated in LPS-induced ALI, while IL-1 $\beta$, IL-6, TNF $\alpha$, IRAK1 and TRAF6 were all upregulated. Subsequent, correlation analysis revealed that the miR-490-3p expression level was negatively correlated to IL-1 $\beta$, IL- 6 , TNF $\alpha$, IRAK1 and TRAF6. Additionally, miR-490-3p overexpression significantly relieved LPS-induced lung epithelial cell injury and the inflammatory response. Mechanistically, miR-490-3p appeared to regulate IRAK1 expression. Overall, the present study revealed that overexpressed miR-490-3p inhibited the IRAK1/TRAF6 pathway, thereby reducing the inflammatory responses in LPS-induced ALI.

miRNAs regulate gene expression by binding to the 3'-UTR of target mRNAs at the post-transcriptional level, thereby modulating its translation (24-26). Previously, miRNAs have been revealed to regulate a variety of inflammatory processes by regulating target genes. For example, miR-29c was revealed to inhibit the production of inflammatory cytokines and reduce the apoptosis rate in Parkinson's disease by targeting specificity protein 1 (27). miR- 485 has been revealed to inhibit the inflammation and multiplication of mesangial cells in vitro by acting on NAPDH oxidase 5 (28). Similarly, multiple miRNAs have been revealed to regulate inflammation in ALI. Meng et al (29) revealed that miR-539-5p relieved sepsis-induced ALI by targeting the protein Rho associated coiled-coil containing protein kinase 1. Yang et al (30) revealed that miR-142a-3p alleviated ALI induced by E. coli lipopolysaccharides by acting on TGF- $\beta$-activated kinase 1 


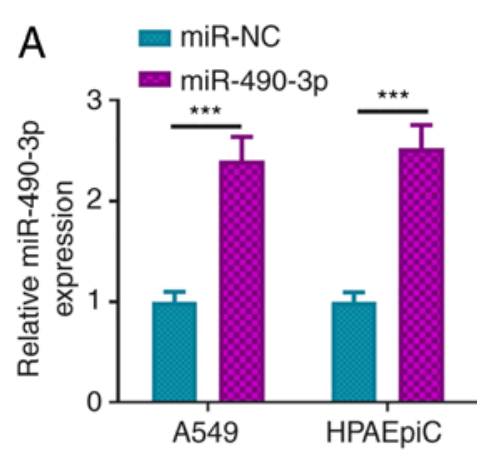

B
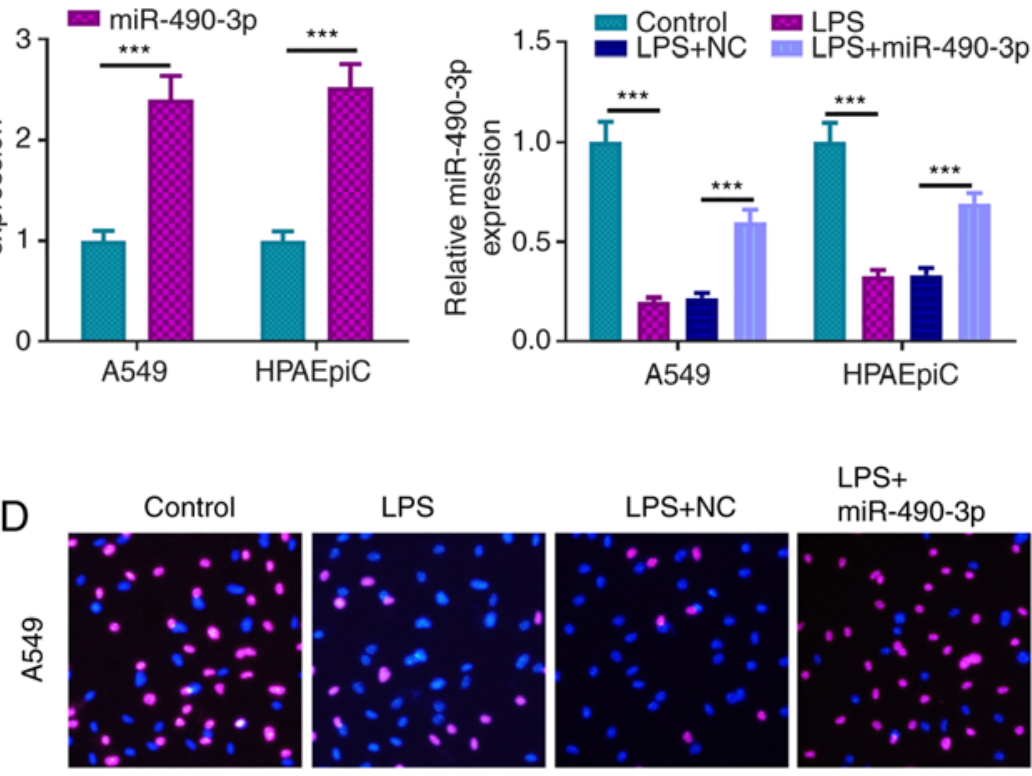

LPS+
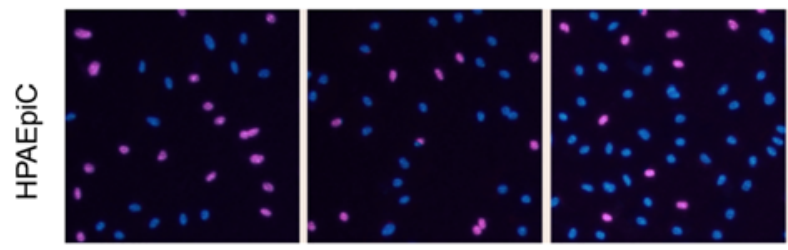

A549

E

है

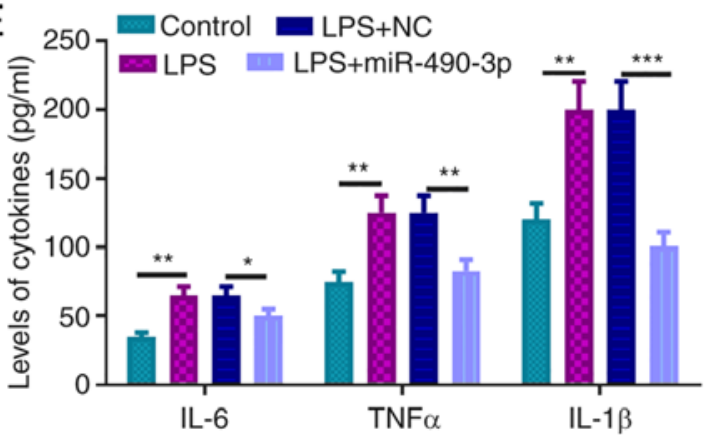
miR-490-3p
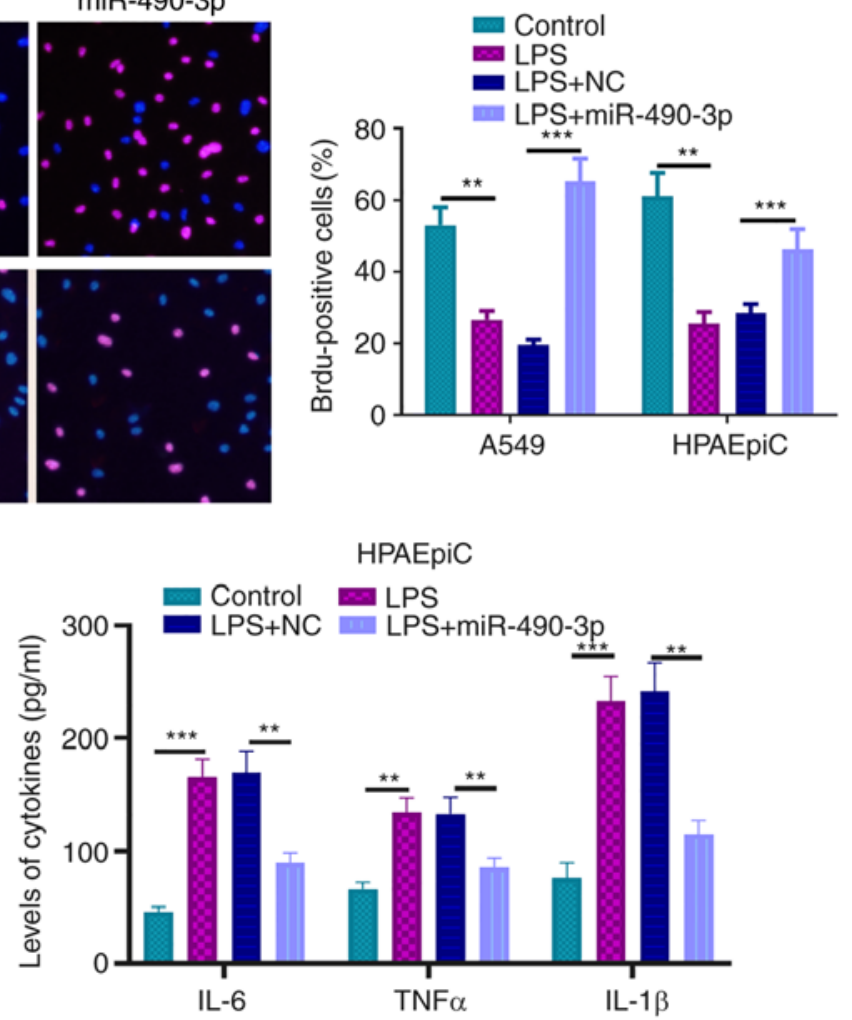

HPAEpiC

Figure 3. miR-490-3p overexpression significantly decreases LPS-induced cell injury and inflammatory response. (A) miR-490-3p expression in A549 and HPAEpiC cells following transfection was determined by RT-qPCR. (B) miR-490-3p relative expression in A549 and HPAEpiC cells following various treatment conditions was determined by RT-qPCR. (C) Cell Counting Kit-8 was used to examine cell proliferation in the four treatment groups after 24 h. (D) BrdU was used to detect cell viability in the four treatment groups. (E) IL-1 $\beta$, IL- 6 and TNF $\alpha$ expression levels in the various treatment groups were monitored by ELISA. ${ }^{*} \mathrm{P}<0.05,{ }^{* *} \mathrm{P}<0.01$ and ${ }^{* * *} \mathrm{P}<0.001$. miR, microRNA; LPS, lipopolysaccharide; RT-qPCR, reverse transcription-quantitative PCR; NC, negative control.

and MAP3K7-binding protein 2. Xie et al (31) claimed that miR-34b-5p aggravated ALI in LPS-induced rat models by targeting progranulin (PGRN), and its knockdown reduced lung inflammation and cell apoptosis. The aforementioned studies have revealed that miRNAs exert protective effects against lung injury via repressing inflammatory responses. Moreover, a number of studies have indicated that attenuating pulmonary inflammation ameliorated acute lung injury in neonatal animal models (32-34). The present research confirmed that miR-490-3p was significantly downregulated in the LPS-induced neonatal ALI rat model. Via in vitro experiments, it was revealed that upregulating miR-490-3p attenuated LPS-induced pulmonary epithelial cell damage, as well as induced inflammatory cytokine expression. Therefore,

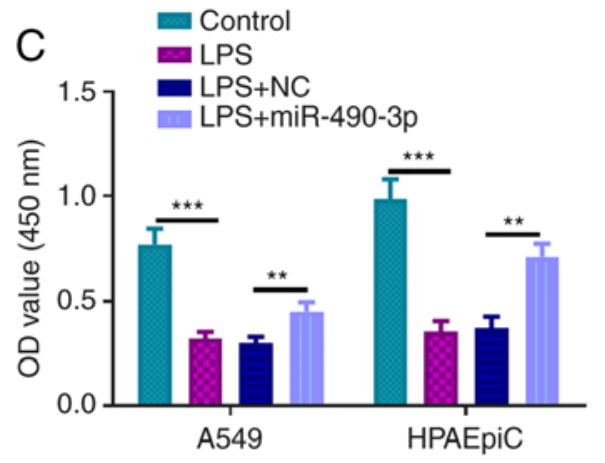



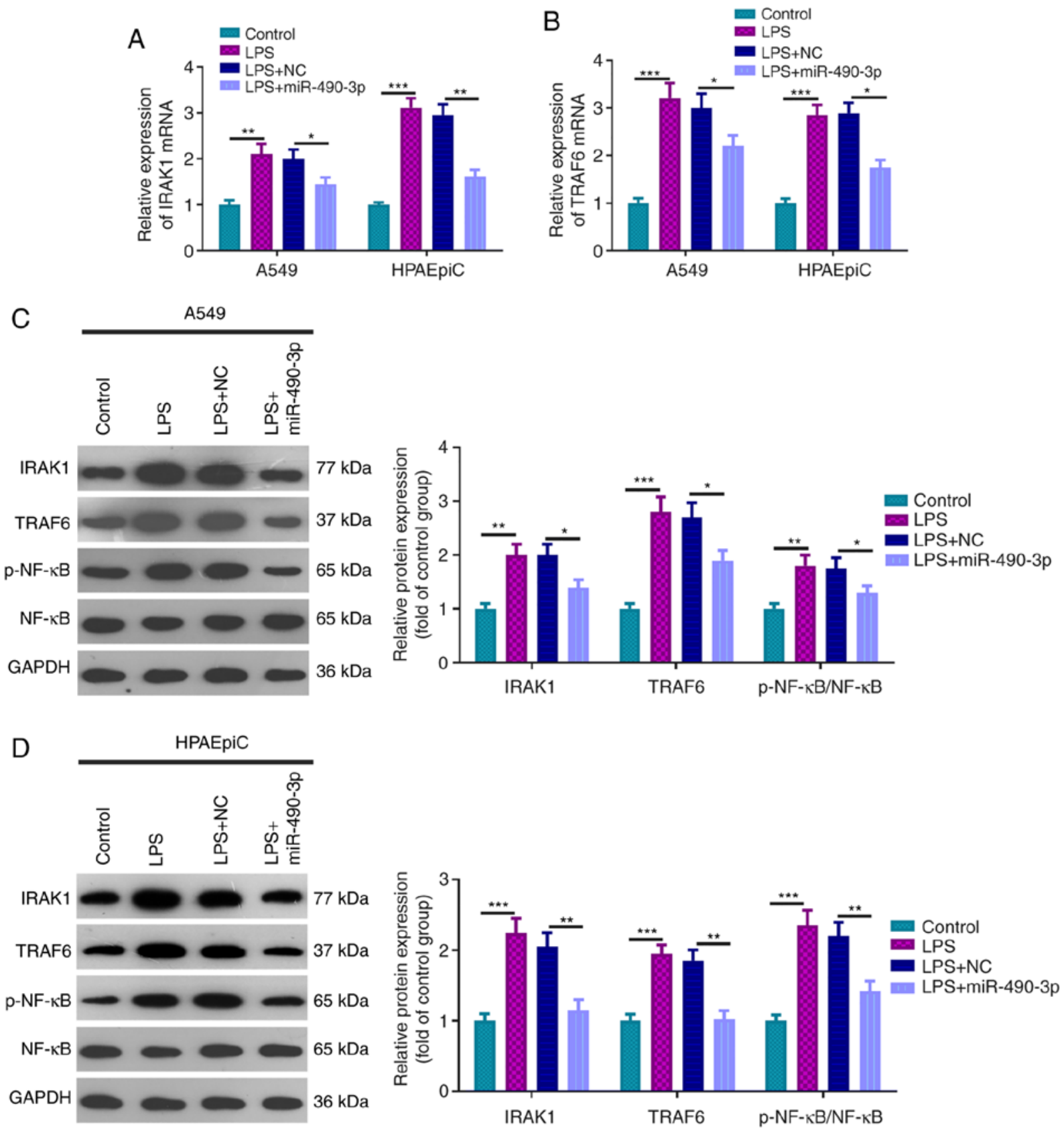

Figure 4. miR-490-3p overexpression restrains IRAK1 and TRAF6 expression. The relative expression levels of (A) IRAK1 and (B) TRAF6 mRNA in the control group, LPS group, LPS + NC group, LPS + miR-490-3p group were verified by reverse transcription-quantitative PCR. Western blot assays were conducted to examine the relative expression levels of IRAK1, TRAF6 and p-NF-kB in the aforementioned treatment groups in (C) A549 and (D) HPAEpiC cells. ${ }^{*} \mathrm{P}<0.05,{ }^{* * *} \mathrm{P}<0.01$ and ${ }^{* * * *} \mathrm{P}<0.001$. miR, microRNA; IRAK1, interleukin 1 receptor associated kinase 1; TRAF6, TNF receptor associated factor 6; LPS, lipopolysaccharide; p-, phosphorylated; $\mathrm{NC}$, negative control.

A

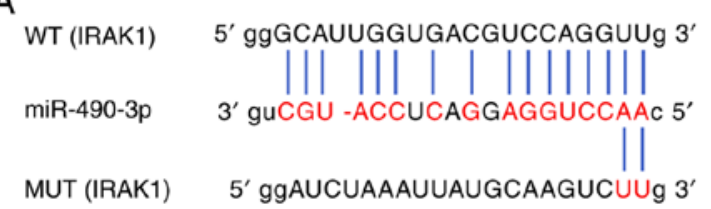

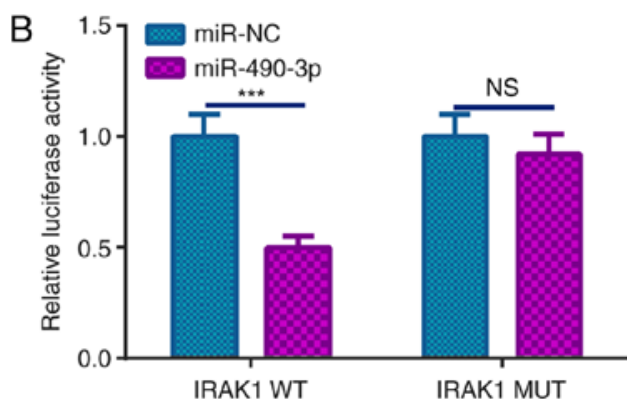

Figure 5. miR-490-3p targets IRAK1. (A) Bioinformatics was used to predict possible binding sites between miR-490-3p and IRAK1. (B) A549 cells were cotransfected with WT-IRAK1, MUT-IRAK1 and miR-490-3p or miR-NC to detect luciferase viability. ${ }^{* * *} \mathrm{P}<0.001$. WT, wild-type; MUT, mutant; NC, negative control; IRAK1, interleukin 1 receptor associated kinase 1; miR, microRNA; NS, not significant. 
Notably, it has been demonstrated that various miRNAs target TLR4 and its downstream signaling molecules IRAK1 and TRAF6 to regulate inflammatory responses. Considering miR-382-3p as an example, it was revealed to inhibit IL-1 $\beta$ induced chondrocyte inflammatory response by directly targeting connexin 43 of the TLR4/MyD88/NF- $\kappa \mathrm{B}$ signaling pathway (39). miR-451 attenuated the inflammation caused by microglial activation and alleviated inflammatory pain by targeting TLR4 (40). Similarly, miRNAs have similar pathway regulation in ALI. For instance, miR-34b-5p targeted PGRN, and its knockdown reduced lung inflammation and apoptosis in an LPS-induced ALI rat model (31). miR-16 impeded ALI by regulating the TLR4/NF- $\kappa$ B pathway and restricting the inflammatory response (41). It can be observed that miRNAs have been revealed to regulate TLR4 signaling pathways through their target genes in diversified inflammations (42). IRAK1, as a key molecule in the TLR4 signaling pathway, is also regulated by miRNAs. For example, miR-146a was revealed to bind to the 3'-UTR of IRAK1 and TRAF6, which attenuated their expression, thereby restricting activation of the inflammatory proteins including NF- $\kappa \mathrm{B}, \mathrm{p} 38$ and ERK1/2 (43). Overexpressing miR-146 reduced IRAK-1 and TRAF6 expression levels, thereby attenuating the inflammatory factors released, and attenuating LPS-induced ALI (44). A more general view is that IRAK-1 exacerbates injury by aggravating the inflammatory response, and it is also targeted by multiple miRNAs. The present study also revealed that miR-490-3p targeted IRAK1 and attenuated its expression.

Overall, the present study indicated that miR-490-3p overexpression distinctly inhibited LPS-induced ALI and inflammatory responses by suppressing the IRAK1/TRAF6 pathway. However, future studies are required to further explore the therapeutic effects of miR-490-3p in ALI in vivo and validate the expression pattern of miR-490-3p in neonates with ALI.

\section{Acknowledgements}

Not applicable.

\section{Funding}

No funding was received.

\section{Availability of data and materials}

The data sets used and analyzed during the present study are available from the corresponding author on reasonable request.

\section{Authors' contribution}

GY conceived, designed and performed the experiments and subsequently wrote the manuscript. YZ conducted the statistical analysis. Both authors read and approved the final manuscript.

\section{Ethics approval and consent to participate}

The present study was approved by the Ethics Review Board of Shanxi Medical University (Taiyuan, China).

\section{Patient consent for publication}

Not applicable.

\section{Competing interests}

The authors declare that they have no competing interests.

\section{References}

1. Chen $\mathrm{H}$, Bai $\mathrm{C}$ and Wang X: The value of the lipopolysaccharideinduced acute lung injury model in respiratory medicine. Expert Rev Respir Med 4: 773-783, 2010.

2. Huang X, Xiu H, Zhang S and Zhang G: The role of macrophages in the pathogenesis of ALI/ARDS. Mediators Inflamm 2018: 1264913, 2018.

3. Liu H, Yu X, Yu S and Kou J: Molecular mechanisms in lipopolysaccharide-induced pulmonary endothelial barrier dysfunction. Int Immunopharmacol 29: 937-946, 2015.

4. Killien EY, Mills B, Watson RS, Vavilala MS and Rivara FP: Morbidity and mortality among critically injured children with acute respiratory distress syndrome. Crit Care Med 47: e112-e119, 2019.

5. Saliminejad K, Khorram Khorshid HR, Soleymani Fard S and Ghaffari SH: An overview of microRNAs: Biology, functions, therapeutics, and analysis methods. J Cell Physiol 234: 5451-5465, 2019.

6. Essandoh K, Li Y, Huo J and Fan GC: MiRNA-mediated macrophage polarization and its potential role in the regulation of inflammatory response. Shock 46: 122-131, 2016.

7. Yao Y, Xu K, Sun Y, Tian T, Shen W, Sun F, Yuan W, Wu H, Chen G, Yuan L, et al: MiR-215-5p inhibits the inflammation injury in septic H9c2 by regulating ILF3 and LRRFIP1. Int Immunopharmacol 78: 106000, 2020. doi: 10.1016/j. intimp.2019.106000.

8. Yan Z, Zang B, Gong X, Ren J and Wang R: MiR-214-3p exacerbates kidney damages and inflammation induced by hyperlipidemic pancreatitis complicated with acute renal injury. Life Sci 241: 117118, 2020.

9. Li P, Yao Y, Ma Y and Chen Y: MiR-150 attenuates LPS-induced acute lung injury via targeting AKT3. Int Immunopharmacol 75: 105794, 2019.

10. Suo T, Chen GZ, Huang Y, Zhao KC, Wang $\mathrm{T}$ and $\mathrm{Hu} \mathrm{K}$ : miRNA-1246 suppresses acute lung injury-induced inflammation and apoptosis via the NF- $\mathrm{BB}$ and $\mathrm{Wnt} / \beta$-catenin signal pathways. Biomed Pharmacother 108: 783-791, 2018.

11. Zhang F, Wu A, Wang Y and Liu J: miR-490-3p functions as a tumor suppressor in glioma by inhibiting high-mobility group AT-hook 2 expression. Exp Ther Med 18: 664-670, 2019.

12. Liu Y, Zhang M, Lou L, Li L, Zhang Y, Chen W, Zhou W, Bai Y and Gao J: IRAK-M Associates with susceptibility to adult-onset asthma and promotes chronic airway inflammation. J Immunol 202: 899-911, 2019.

13. Sharma A, Maurya CK, Arha D, Rai AK, Singh S, Varshney S, Schertzer JD and Tamrakar AK: Nod1-mediated lipolysis promotes diacylglycerol accumulation and successive inflammation via PKC $\delta$-IRAK axis in adipocytes. Biochim Biophys Acta Mol Basis Dis 1865: 136-146, 2019.

14. Zhang QB, Qing YF, Yin CC, Zhou L, Liu XS, Mi QS and Zhou JG: Mice with miR-146a deficiency develop severe gouty arthritis via dysregulation of TRAF 6, IRAK 1 and NALP3 inflammasome. Arthritis Res Ther 20: 45, 2018.

15. Wang J, Wu J, Cheng Y, Jiang Y and Li G: Over-expression of microRNA-223 inhibited the proinflammatory responses in Helicobacter pylori-infection macrophages by down-regulating IRAK-1. Am J Transl Res 8: 615-622, 2016.

16. Shekarforoush S, Fatahi Z and Safari F: The effects of pentobarbital, ketamine-pentobarbital and ketamine-xylazine anesthesia in a rat myocardial ischemic reperfusion injury model. Lab Anim 50: 179-184, 2016.

17. Francischi JN, Frade TIC, Almeida MPA, Queiroz BFG and Bakhle YS: Ketamine-xylazine anaesthesia and orofacial administration of substance P: A lethal combination in rats. Neuropeptides 62: 21-26, 2017. 
18. Kocaturk H, Bedir F, Altay MS, Bakan E, Suleyman B, Yazici GN, Sunar M, Suleyman Z and Suleyman H: The effect of desloratadine on ischemia reperfusion induced oxidative and inflammatory renal injury in rats. Ren Fail 42: 531-538, 2020.

19. Livak KJ and Schmittgen TD: Analysis of relative gene expression data using real-time quantitative PCR and the 2(- $\Delta \Delta$ C(T)) Method. Methods 25: 402-408, 2001.

20. Li JH, Liu S, Zhou H, Qu LH and Yang JH: starBase v2.0: Decoding miRNA-ceRNA, miRNA-ncRNA and protein-RNA interaction networks from large-scale CLIP-Seq data. Nucleic Acids Res 42 (D1): D92-D97, 2014.

21. Chakraborty M, McGreal EP and Kotecha S: Acute lung injury in preterm newborn infants: Mechanisms and management. Paediatr Respir Rev 11: 162-170, quiz 170, 2010.

22. Cheng N, Liang Y, Du X and Ye RD: Serum amyloid A promotes LPS clearance and suppresses LPS-induced inflammation and tissue injury. EMBO Rep 19: e45517, 2018.

23. Wang $\mathrm{Q}$ and Xiao L: Isochlorogenic acid $\mathrm{A}$ attenuates acute lung injury induced by LPS via Nf- $\kappa \mathrm{B} / \mathrm{NLRP} 3$ signaling pathway. Am J Transl Res 11: 7018-7026, 2019.

24. Yang H, Song Z and Hong D: CRBN knockdown mitigates lipopolysaccharide-induced acute lung injury by suppression of oxidative stress and endoplasmic reticulum (ER) stress associated NF- $\kappa$ B signaling. Biomed Pharmacother 123: 109761, 2020.

25. Lee HM, Kim TS and Jo EK: MiR-146 and miR-125 in the regulation of innate immunity and inflammation. BMB Rep 49: 311-318, 2016.

26. Haneklaus M, Gerlic M, O'Neill LA and Masters SL: miR-223: Infection, inflammation and cancer. J Intern Med 274: 215-226, 2013.

27. Wang R, Yang Y, Wang H, He Y and Li C: MiR-29c protects against inflammation and apoptosis in Parkinson's disease model in vivo and in vitro by targeting SP1. Clin Exp Pharmacol Physiol 47: 372-382, 2020

28. Wu J, Lu K, Zhu M, Xie X, Ding Y, Shao X, Chen Y, Liu J, Xu M, $\mathrm{Xu}$ Y, et al: $\mathrm{miR}-485$ suppresses inflammation and proliferation of mesangial cells in an in vitro model of diabetic nephropathy by targeting NOX5. Biochem Biophys Res Commun 521: 984-990, 2020 .

29. Meng L, Cao H, Wan C and Jiang L: MiR-539-5p alleviates sepsis-induced acute lung injury by targeting ROCK1. Folia Histochem Cytobiol 57: 68-178, 2019.

30. Yang Y, Yang C, Guo Y-F, Liu P, Guo S, Yang J, Zahoor A, Shaukat A and Deng G: MiR-142a-3p alleviates Escherichia coli derived lipopolysaccharide-induced acute lung injury by targeting TAB2. Microb Pathog 136: 103721, 2019.

31. Xie W, Lu Q, Wang K, Lu J, Gu X, Zhu D, Liu F and Guo Z: miR-34b-5p inhibition attenuates lung inflammation and apoptosis in an LPS-induced acute lung injury mouse model by targeting progranulin. J Cell Physiol 233: 6615-6631, 2018.

32. Cheng K, Yang A, Hu X, Zhu D and Liu K: Curcumin attenuates pulmonary inflammation in lipopolysaccharide induced acute lung injury in neonatal rat model by activating peroxisome proliferator-activated receptor $\gamma$ (PPAR $\gamma)$ pathway. Med Sci Monit 24: 1178-1184, 2018
33. Duan Q, Jia Y, Qin Y, Jin Y, Hu H and Chen J: Narciclasine attenuates LPS-induced acute lung injury in neonatal rats through suppressing inflammation and oxidative stress. Bioengineered 11: 801-810, 2020

34. Wang X, Zhang C, Chen C, Guo Y, Meng X and Kan C: Allicin attenuates lipopolysaccharide-induced acute lung injury in neonatal rats via the PI3K/Akt pathway. Mol Med Rep 17: 6777-6783, 2018

35. Yang D, Li S, Duan X, Ren J, Liang S, Yakoumatos L, Kang Y, Uriarte SM, Shang J, Li W, et al: TLR4 induced Wnt3a-Dvl3 restrains the intensity of inflammation and protects against endotoxin-driven organ failure through GSK3 $\beta / \beta$-catenin signaling. Mol Immunol 118: 153-164, 2020.

36. Zhang B, Zeng M, Li M, Kan Y, Li B, Xu R, Wu Y, Wang S, Zheng $X$ and Feng $W$ : Protopine protects mice against LPS-induced acute kidney injury by inhibiting apoptosis and inflammation via the TLR4 signaling pathway. Molecules 25: 25, 2019.

37. Yan S, Wang P, Wang J, Yang J, Lu H, Jin C, Cheng M and Xu D: Long non-coding RNA HIX003209 promotes inflammation by sponging miR-6089 via TLR4/NF- $\mathrm{BB}$ signaling pathway in rheumatoid arthritis. Front Immunol 10: 2218, 2019.

38. Giriwono PE, Shirakawa H, Ohsaki Y, Sato S, Aoyama Y, Ho HJ, Goto T and Komai M: Geranylgeraniol suppresses the expression of IRAK1 and TRAF6 to inhibit NF- $\kappa \mathrm{B}$ activation in lipopolysaccharide-induced inflammatory responses in human macrophage-like cells. Int J Mol Sci 20: 20, 2019.

39. Lei J,Fu Y,Zhuang Y,Zhang K and Lu D: miR-382-3p suppressed IL-1 $\beta$ induced inflammatory response of chondrocytes via the TLR4/MyD88/NF- $\kappa$ B signaling pathway by directly targeting CX43. J Cell Physiol 234: 23160-23168, 2019.

40. Sun $X$ and Zhang H: miR-451 elevation relieves inflammatory pain by suppressing microglial activation-evoked inflammatory response via targeting TLR4. Cell Tissue Res 374: 487-495, 2018.

41. Yang Y, Yang F, Yu X, Wang B, Yang Y, Zhou X, Cheng R, Xia S and Zhou X: miR-16 inhibits NLRP3 inflammasome activation by directly targeting TLR4 in acute lung injury. Biomed Pharmacother 112: 108664, 2019.

42. Xie MY, Hou LJ, Sun JJ, Zeng B, Xi QY, Luo JY, Chen T and Zhang YL: Porcine milk exosome MiRNAs attenuate LPS-induced apoptosis through inhibiting TLR4/NF- $\kappa$ B and p53 pathways in intestinal epithelial cells. J Agric Food Chem 67: 9477-9491, 2019.

43. Zeng R, Xu H, Liu Y, Du L, Duan Z, Tong J, He Y, Chen Q, Chen X and Li M: miR-146a inhibits biofilm-derived cutibacterium acnes-induced inflammatory reactions in human keratinocytes. J Invest Dermatol 139: 2488-2496.e4, 2019.

44. Zeng Z, Gong H, Li Y, Jie K, Ding C, Shao Q, Liu F, Zhan Y, Nie C, Zhu W, et al: Upregulation of miR-146a contributes to the suppression of inflammatory responses in LPS-induced acute lung injury. Exp Lung Res 39: 275-282, 2013.

(i) $\odot$ This work is licensed under a Creative Commons Attribution-NonCommercial-NoDerivatives 4.0 International (CC BY-NC-ND 4.0) License. 